\title{
God gives IL-19 with both hands: anti-inflammatory but pro-angiogenic
}

Xiaoxiang Yan $^{1,3}$, Motoaki Sano ${ }^{2,3}$

1 Department of Cardiology, Ruijin Hospital, Shanghai Jiao Tong University School of Medicine, Shanghai, China. ${ }^{2}$ Department of Cardiology, Keio University School of Medicine, Tokyo, Japan

${ }^{3}$ Address for correspondence: Dr. Motoaki Sano, Department of Cardiology, Keio University School of Medicine 35 Shinanomachi, Shinjuku-ku, Tokyo 1608582, Japan. Tel: +81-(3)-3353-1211, Ext. 61484; Fax: +81-(3)-5363-3875; Email: msano@a8.keio.jp or Dr. Xiaoxiang Yan, Department of Cardiology, Ruijin Hospital, Shanghai Jiao Tong University School of Medicine, Shanghai 200025, People's Republic of China. Tel: +86-(21)-6437-0045, Ext. 610910; E-mail: cardexyanxx@hotmail.com

Keywords: Ischemia; Inflammation; Interleukin-19; Angiogenesis

Word count of manuscript: 2360 words 
Peripheral artery diseases (PAD) and coronary artery diseases (CAD) are caused by atherosclerosis and thrombosis. As a complication of PAD, critical limb ischemia (CLI) causes non-healing ulcers and pain on walking. Patients with CLI are generally treated with a combination of risk factor modifiers, such as statins, anti-platelet drugs, and angioplasty. These treatments, however, occasionally do not restore blood flow to a degree that allows for normal tissue function [1]. Therapeutic angiogenesis offers a promising approach to promoting new vessel growth and re-establishing sufficient blood flow in the limb. Molecules which cannot only limit tissue inflammation but also increase capillary density, collateral formation and perfusion potentially salvage ischemic limbs and can lead to new therapies for CLI.

Several homeostatic and pathological processes depend on the development of new vasculature, particularly of new capillaries from endothelial cells (ECs) arising from existing vessels [2]. Angiogenesis is initiated in a hypoxic environment (often encountered in tissue inflammation) and is likely

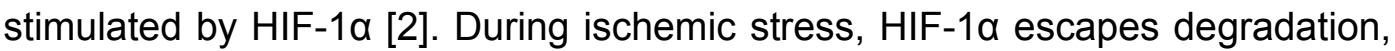
binds to the HIF-1 $\beta$ subunit and activates target genes involved in angiogenesis [3]. Human VEGF-A has at least six isoforms: 121, 145, 165, 183, 189, and 204 due to the alternative splicing of a single gene. Of these, VEGF-A165 and VEGF-A121 are the most potent proangiogenic isoforms. VEGF-A165a and VEGF-A165b isoforms have an opposite effect on angiogenesis. VEGF-A (VEGF-A165a), an essential angiogenic factor, has been shown in several in vivo models to induce a strong concomitant angiogenic cascade with HIF-1 [4]. Upon stimulation with macrophage and T-lymphocyte-derived VEGF-A (VEGF-A165a), ECs produce monocyte chemoattractant protein (MCP)-1, which attracts monocytes and increases the permeability of the endothelial layer to facilitate cell migration into the tissues [5]. There, monocytes mature into macrophages, producing cytokines and growth factors.

Besides hypoxic stimuli, chronic infection, cigarette smoking, free radicals, hypertension, and diabetes have all been implicated as causes in the 
activation of ECs [2]. Increased shear stress can also stimulate quiescent ECs lining the vascular wall. Intracellular signaling activates genes involved in the induction of transcription factors that regulate shear stress-mediated effects [6]. Under hypercholesterolemia, these events stimulate MCP-1 release and expression of the MCP-1 receptor, CCR2, and several adhesion molecules, particularly P-selectin, E-selectin, VCAM-1 and ICAM-1. This allows leukocyte adhesion, migration and activation in local tissue [6].

All in all, both acute ischemia and traditional risk factors lead to increased infiltration of local tissue leukocytes and the expression of inflammatory cytokines with pro-angiogenic and anti-angiogenic properties balancing the response. Other than the better known angiogenic proteins like VEGF-A, FGF, and CXCL1, many pro-inflammatory cytokines such as IL-1a, IL6 , IL-8, and IL-18 play important roles in increasing EC migration, proliferation, tube formation, and vascularity in vivo [7-9]. On the other hand, the effect of anti-inflammatory interleukins on EC in initiating angiogenesis is less clear.

IL-19 was discovered in 2000 as a member of the IL-10 superfamily, which includes IL-10, IL-19, IL-20, IL-22, IL-24, IL-26, IL-28, and IL-29. IL-19 skews the immune response towards a $\mathrm{T}_{\mathrm{H}} 2$ type, resulting in more IL-4producing and fewer IFN- $\gamma-$ producing cells [10]. Most findings about IL-19's role in vascular pathophysiology have been reported by Michael V. Autieri's group [11-19]. Unlike other Th2 interleukins, IL-19 appears to stimulate angiogenesis. Although IL-19 shares $21 \%$ amino acid identity with IL-10, IL-19 acts differently from IL-10 in the creation of new blood vessels. IL-19 promotes, while IL-10 suppresses angiogenesis $[10,17,20]$. IL-19 is primarily produced by monocytes, and its expression can be induced in vascular smooth muscle cells (VSMCs) and ECs by inflammatory stimuli, including T-cell-conditioned media, IFN- $\gamma$, platelet-derived growth factor (PDGF), basic fibroblast growth factor (bFGF), vascular endothelial growth factor-A (VEGF-A), oxidized LDL (ox-LDL) and TNF- $\alpha[17,19]$. IL-19 uses a receptor complex composed of the IL-20R1 and IL-20R2 chains. This signal activates monocytes in an autocrine and paracrine fashion. Immunohistochemistry revealed that IL-19 was highly 
expressed in VSMC, EC, and CD45+ leukocytes in atherosclerotic plaques from human and mouse [12]. The same phenomenon was found in the mouse model of limb ischemia $[17,18]$.

Angiogenesis requires the recruitment of smooth muscle cells (SMCs) to stabilize the nascent vessel. Platelet-derived growth factor (PDGF) attracts lining mesenchymal cells to the new vessel, where they respond to transforming growth factor- $\beta$ (TGF- $\beta$ ) signaling by differentiating into vascular SMCs (VSMCs). If ECs and VSMCs continue to bind tightly, TGF expression is sustained and cell proliferation for the formation of the vessel is mutually inhibited [21]. It seems that IL-19 exerts opposing effects on VSMCs and ECs. In VSMCs, IL-19 decreases the expression of pro-inflammatory and proliferative genes in an NF-kB-independent mechanism. In both FBS-stimulated VSMCs and TNF- $\alpha$-stimulated human coronary artery ECs, IL-19 suppresses proinflammatory gene expression by reducing HuR-promoted mRNA stability [11, 14]. IL-19 might also reduce the activation of p44/42 and p38 MAPKs in stimulated VSMCs by inducing SOCS5 and inhibiting signal transduction. IL-19 evokes a rapid STAT3 activation and increases expression of suppressor of SOCS5, a STAT-responsive gene, at mRNA and protein levels in VSMCs. SOCS5 interacts with both p44/42 and p38 MAPKs in IL-19-treated human VSMCs [19]. IL-19 inhibits the activation of cellular motility proteins, including myosin light chain (MLC), cofilin, Hsp70, and the monomeric G proteins Rac1 and RhoA, resulting in decreased VSMC motility [16]. Since motility of VSMC is an essential step for angiogenesis, IL-19 may negatively affect angiogenesis during ischemia. Despite impairing proliferation in VSMCs, IL-19 stimulates proliferation, migration and angiogenesis in ECs by activating STAT3, Rac1, and MAPK p44/42 [17]. These functions are independent of bFGF and VEGF-A and are IL-19-specific, as anti-IL-20 receptor antibodies significantly reduce IL19-driven EC migration [22]. IL-19 induces expression of CXCL1, CXCL8 (IL-8), and HGF in human microvascular ECs but not FGF, VEGF-A, and HIF-1a [17]. CXCL1 and IL-8 are chemokines of the CXC family and not only chemotactic for leukocytes, but also regulate EC motility, proliferation, and viability, and both 
possess angiogenic properties [23]. HGF potently triggers cell proliferation of ECs, and is angiogenic in ischemic mouse and rabbit hind limbs [24]. IL-19 also decreases leukocyte-EC interactions by reducing EC adhesion molecule mRNA stability [14].

In this issue of the Journal of Molecular and Cellular Cardiology, Richards and colleagues show that IL-19 can increase perfusion of ligated murine hind limbs through various mechanisms and effects on multiple target cells [18]. They demonstrate that injection of IL-19 increases, and lack of IL-19 decreases, reperfusion and angiogenesis in ischemic hind limbs. Potential mechanisms include direct angiogenic effects on ECs, macrophage M2 polarization and VEGF-A (VEGF-A165a) induction, and indirect effects by reducing $\mathrm{IL}-12$ expression in macrophages. This implies a paracrine function of IL-19 in EC-macrophage cross-talk during angiogenesis.

Macrophages can be classified as M1 or M2 based on their activation state. The anti-inflammatory or "wound healing" pathway of M2 is typically induced by IL-10, IL-4, and IL-13 [25]. The authors found that IL-19 shifts macrophage polarization towards M2 [18]. M2 macrophages secrete EC growth and angiogenic factors such as VEGF-A (VEGF-A165a) and CXCL1, which are implicated in wound repair and neovascularization [25]. In this regard, IL-19 is similar to the Th2 interleukins IL-4 and IL-13. IL-19 seems to have no effect on macrophage infiltration in the ischemic hind limbs model, while it suppresses macrophage infiltration in the atherosclerotic model [12]. This may be due to the different disease model: atherosclerosis is a chronic low-grade inflammation, while the ischemic hind limbs model features acute severe inflammation. IL-19 is not capable of inhibiting the overwhelming macrophage infiltration in ischemic hind limbs.

Besides hypoxia, numerous cytokines including IL-19 can stimulate VEGF-A (VEGF-A165a) production in macrophages [18], which acts as a primary driver of neovascularization by inducing angiogenic gene expression, proliferation and differentiation of ECs. Interestingly, IL-19 does not induce VEGF-A (VEGF-A165a) expression in cultured human microvascular ECs, 
suggesting complex and cell-specific effects of IL-19 on angiogenic and inflammatory responses. Thus, the angiogenic properties of IL-19 are brought about by a polarization shift towards M2 and an induction of VEGF-A (VEGFA165a) in macrophages.

IL-12 is a potent pro-inflammatory cytokine expressed in leukocytes, driving the Th1 pro-inflammatory response and exerting a powerful antiangiogenic function [26]. Researchers demonstrated that IL-19 inhibits IL-12 expression in macrophages [18], which might explain the pro-angiogenic effect of IL-19. This effect seems to be indirect, since the IL-12 receptor is not expressed on ECs.

In conclusion, the current study and previously published reports by Autieri's group support the intriguing possibility that IL-19 can modulate inflammation outside of the immune system, and directly exerts effects on the vasculature by controlling EC and/or smooth muscle cell phenotype and responses to inflammation and ischemia. IL-19's direct pro-angiogenic effects on ECs, an increase in M2-polarized macrophages and inhibition of antiangiogenic cytokine expression in macrophages are proposed mechanisms for pro-angiogenic property of IL-19. Apart from inflammation, several other processes contribute to angiogenesis: cell-to-extracellular matrix interactions, vascular wall maturation and basal lamina modifications [27]. Future studies are necessary to delineate how IL-19 regulates these processes to form functional vessels in ischemic tissues.

\section{Acknowledgements}

This work was supported by a PRESTO (chronic inflammation) grant from the Japan Science and Technology Agency (to M.S.)

\section{Disclosure}

None. 


\section{References}

[1] Tanaka M, Taketomi K, Yonemitsu Y. Therapeutic angiogenesis: recent and future prospects of gene therapy in peripheral artery disease. Curr Gene Ther. 2014; 14:300-8.

[2] Jaipersad AS, Lip GY, Silverman S, Shantsila E. The role of monocytes in angiogenesis and atherosclerosis. J Am Coll Cardiol. 2014;63:1-11.

[3] Kimura H, Esumi H. Reciprocal regulation between nitric oxide and vascular endothelial growth factor in angiogenesis. Acta Biochim Pol. 2003;50:49-59.

[4] Kuwahara F, Kai H, Tokuda K, Shibata R, Kusaba K, Tahara N, et al. Hypoxia-inducible factor-1alpha/vascular endothelial growth factor pathway for adventitial vasa vasorum formation in hypertensive rat aorta. Hypertension. 2002;39:46-50.

[5] Hong KH, Ryu J, Han KH. Monocyte chemoattractant protein-1-induced angiogenesis is mediated by vascular endothelial growth factor-A. Blood. 2005;105:1405-7.

[6] Pamukcu B, Lip GY, Devitt A, Griffiths H, Shantsila E. The role of monocytes in atherosclerotic coronary artery disease. Ann Med. 2010;42:394-403.

[7] Huang S, Mills L, Mian B, Tellez C, McCarty M, Yang XD, et al. Fully humanized neutralizing antibodies to interleukin-8 (ABX-IL8) inhibit angiogenesis, tumor growth, and metastasis of human melanoma. Am J Pathol. 2002;161:125-34.

[8] Park CC, Morel JC, Amin MA, Connors MA, Harlow LA, Koch AE. Evidence of IL-18 as a novel angiogenic mediator. J Immunol. 2001;167:1644-53.

[9] Salven P, Hattori K, Heissig B, Rafii S. Interleukin-1alpha promotes angiogenesis in vivo via VEGFR-2 pathway by inducing inflammatory cell VEGF synthesis and secretion. FASEB J. 2002;16:1471-3.

[10] Commins S, Steinke JW, Borish L. The extended IL-10 superfamily: IL-10, IL-19, IL-20, IL-22, IL-24, IL-26, IL-28, and IL-29. J Allergy Clin Immunol. 2008;121:1108-11.

[11] Cuneo AA, Herrick D, Autieri MV. II-19 reduces VSMC activation by regulation of $m R N A$ regulatory factor HuR and reduction of mRNA stability. $J$ Mol Cell Cardiol. 2010;49:647-54. 
[12] Ellison S, Gabunia K, Kelemen SE, England RN, Scalia R, Richards JM, et al. Attenuation of experimental atherosclerosis by interleukin-19. Arterioscler Thromb Vasc Biol. 2013;33:2316-24.

[13] Ellison S, Gabunia K, Richards JM, Kelemen SE, England RN, Rudic D, et al. IL-19 reduces ligation-mediated neointimal hyperplasia by reducing vascular smooth muscle cell activation. Am J Pathol. 2014;184:2134-43.

[14] England RN, Preston KJ, Scalia R, Autieri MV. Interleukin-19 decreases leukocyte-endothelial cell interactions by reduction in endothelial cell adhesion molecule mRNA stability. Am J Physiol Cell Physiol. 2013;305:C255-65.

[15] Gabunia K, Ellison SP, Singh H, Datta P, Kelemen SE, Rizzo V, et al. Interleukin-19 (IL-19) induces heme oxygenase-1 (HO-1) expression and decreases reactive oxygen species in human vascular smooth muscle cells. $J$ Biol Chem. 2012;287:2477-84.

[16] Gabunia K, Jain S, England RN, Autieri MV. Anti-inflammatory cytokine interleukin-19 inhibits smooth muscle cell migration and activation of cytoskeletal regulators of VSMC motility. Am J Physiol Cell Physiol. 2011;300:C896-906.

[17] Jain S, Gabunia K, Kelemen SE, Panetti TS, Autieri MV. The antiinflammatory cytokine interleukin 19 is expressed by and angiogenic for human endothelial cells. Arterioscler Thromb Vasc Biol. 2011;31:167-75.

[18] Richards J, Gabunia K, Kelemen SE, Kako F, Choi ET, Autieri MV. Interleukin-19 increases Angiogenesis in ischemic hind limbs by Direct Effects on both Endothelial Cells and Macrophage Polarization. J Mol Cell Cardiol. 2014;http://dx.doi.org/10.1016/j.yjmcc.2014.11.002.

[19] Tian Y, Sommerville LJ, Cuneo A, Kelemen SE, Autieri MV. Expression and suppressive effects of interleukin-19 on vascular smooth muscle cell pathophysiology and development of intimal hyperplasia. Am $\mathrm{J}$ Pathol. 2008;173:901-9.

[20] England RN, Autieri MV. Anti-inflammatory effects of interleukin-19 in vascular disease. Int J Inflam. 2012;2012:253583.

[21] ten Dijke P, Arthur HM. Extracellular control of TGFbeta signalling in vascular development and disease. Nat Rev Mol Cell Biol. 2007;8:857-69. 
[22] Sakurai N, Kuroiwa T, Ikeuchi H, Hiramatsu N, Maeshima A, Kaneko Y, et al. Expression of IL-19 and its receptors in RA: potential role for synovial hyperplasia formation. Rheumatology (Oxford). 2008;47:815-20.

[23] Zaja-Milatovic S, Richmond A. CXC chemokines and their receptors: a case for a significant biological role in cutaneous wound healing. Histol Histopathol. 2008;23:1399-407.

[24] Morishita R, Nakamura S, Hayashi S, Taniyama Y, Moriguchi A, Nagano T, et al. Therapeutic angiogenesis induced by human recombinant hepatocyte growth factor in rabbit hind limb ischemia model as cytokine supplement therapy. Hypertension. 1999;33:1379-84.

[25] Jetten N, Verbruggen S, Gijbels MJ, Post MJ, De Winther MP, Donners MM. Anti-inflammatory M2, but not pro-inflammatory M1 macrophages promote angiogenesis in vivo. Angiogenesis. 2014;17:109-18.

[26] Strasly M, Cavallo F, Geuna M, Mitola S, Colombo MP, Forni G, et al. IL-12 inhibition of endothelial cell functions and angiogenesis depends on lymphocyte-endothelial cell cross-talk. J Immunol. 2001;166:3890-9.

[27] Hoefer IE, van Royen N, Rectenwald JE, Deindl E, Hua J, Jost M, et al. Arteriogenesis proceeds via ICAM-1/Mac-1- mediated mechanisms. Circ Res. 2004;94:1179-85. 